\title{
Cambios poblacionales de una comunidad de aves esteparias en una localidad del centro de España en un periodo de 35 años.
}

\section{Population changes of a steppe bird community in central Spain over 35 years.}

Tomás Santos ${ }^{1 *}$

?

\section{Resumen}

Se analizan los cambios interanuales de una comunidad reproductora de aves esteparias en un área de cultivos cerealistas localizada en el centro de España (Sepúlveda, provincia de Segovia). Los datos proceden de ocho itinerarios en los que se registraron los usos de las parcelas agrícolas (cuatro sustratos: pasto, barbecho, arado y mies) y se censaron las aves en las primaveras de 1984, 2015, 2016 y 2019, asignando las observaciones a uno de los sustratos agrarios o a las lindes. La estructura del paisaje agrícola no varió en el periodo considerado, pero los usos agrarios variaron entre años, principalmente como resultado de cambios en las superficies aradas y sembradas. Se encontró un aumento de la riqueza de especies de aves y cambios significativos en la densidad de varias de ellas, con un descenso muy acusado de la calandria común, y tendencias temporales menos claras en otras especies. La variación entre sustratos de la densidad y del índice de electividad de Ivlev muestra que los pastos y lindes beneficiaron a una mayoría de especies, mientras que arados y barbechos tuvieron efectos positivos sobre una única especie (la terrera común y el pardillo, respectivamente), y las mieses fueron rechazadas o usadas en función de su disponibilidad. Como conclusión, se hace hincapié en la importancia de los elementos

\footnotetext{
1 Universidad Complutense.

Departamento de Biodiversidad, Ecología y Evolución, Facultad de Ciencias Biológicas.

28040 Madrid, España

Calle Marqués de Lozoya 23, 28007 Madrid

*Correspondencia autor: tsantos@ucm.es
} 
estables del paisaje agrícola (pastos y lindes) para las poblaciones reproductoras de aves esteparias.

Palabras clave: estepas cerealistas, aves esteparias, sustratos agrarios, pastos, lindes, alaúdidos.

\begin{abstract}
The inter-annual changes in a breeding community of steppe birds are analysed in an area used for growing cereal crops in central Spain (Sepúlveda, province of Segovia). The data come from eight routes in which the uses (pasture, fallow, ploughed land and cornfield substrates) of the agricultural plots were recorded, and the birds censused during the spring of 1984, 2015, 2016 and 2019, assigning the observations to one of the four agricultural substrates or to field borders. The structure of the agricultural landscape did not change, but the agricultural uses varied from one year to another, mainly as a result of changes in ploughed and cornfield areas. An increase was found in bird richness and significant changes in the density of several species, with significant declines in the calandra lark, and less clear temporal trends in other species. The variation between substrates with regard to density and Ivlev's electivity index showed that pastures and field borders benefited a majority of species, while ploughed and fallow fields each had positive effects on a single species (the greater short-toed lark and the common linnet, respectively). Cornfields were rejected, or used according to their availability. In conclusion, the importance of stable elements in agricultural landscapes (pastures and field borders) for breeding populations of steppe birds is emphasised.
\end{abstract}

Keywords: cereal steppes, steppe birds, agricultural substrates, pastures, field borders, larks.

\title{
Laburpena
}

Espainia erdialdean (Sepulveda, Segovia) kokatutako zereal-laborantza eremu bateko estepa-hegaztien ugaltze-komunitate batean urterik urte gertatutako aldaketak aztertu dira. Datuak zortzi ibilbidetan jaso dira. Ibilbide horietan nekazal-lursailen erabilerari buruzko informazioa jaso zen (lau substratu: larrea, lurgorria, goldatua eta uzta) eta 1984, 2015, 2016 eta 2019 urteetako udaberrian zentsatu ziren hegaztiak, behaketa bakoitza lotuta lau substratuetako batekin edo mugakoekin. Aztergai izan zen denbora tartean nekazalpaisaiaren egitura ez zen aldatu baina bai lursailen erabilera urterik urte, batez ere goldatutako eta ereindako eremuen ondorioz. Ikusi zen hegazti espezieen aberastasuna handitu egin zela eta horietako batzuen dentsitatean aldaketa esanguratsuak gertatu zirela: kalandriaren dentsitatea nabarmen behera eta beste espezie batzuetan denbora tarteka joera ez hain esanguratsuak.

Dentsitate substratuen arteko aldakuntzak eta Ivlev-en elektibitate indize aldakuntzak agerian uzten dute larreek eta mugek espezie gehienei onurak dakarzkiela; ordea, espezie bakar baten hobekuntza behatu da goldatuta eta lurgorrian dauden lursailetan (txoriandre 
arrunta eta txoka arrunta hurrenez hurren). Uztak beren eskuragarritasunaren arabera erabili edo baztertu ziren. Ondorio bezala, garrantzitsutzat jotzen da estepa-hegatientzako nekazal-paisaian egitura egonkorrak (larreak eta mugak) egotea.

Gako hitzak: zereal-estepa, estepa-hegaztiak, nekazal-substratuak, larreak, mugak, alaudidoak.

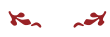

\section{Introducción}

Las aves esteparias europeas, en origen asociadas a regiones y hábitats más bien restringidos, han pasado, tras siglos de incremento de sus áreas de distribución y poblaciones (Santos y Suárez, 2005; Suárez et al., 2009), a una situación de declive que para muchas especies ha llegado a ser crítica (Tucker y Heath, 1994; Donald et al., 2001b; Madroño et al., 2004; Escandell y Escudero, 2020). Dicho incremento fue el resultado de la adaptación a los hábitats de origen humano generados por la actividad agropecuaria y, en particular, a los cultivos de cereal (Bernis, 1972; Suárez, 2004). Sin embargo, las últimas décadas han visto un descenso continuado de estas aves como resultado de la pérdida de diversidad de los paisajes agrícolas y el uso creciente de biocidas, además de otros cambios, como el abandono de los cultivos menos productivos y la disminución del pastoreo extensivo (Robinson y Sutherland, 2002; Benton et al., 2003; Suárez, 2004; Gregory et al., 2005; EEA, 2010; European Commission, 2020).

La agricultura se extiende sobre un $47 \%$ del territorio de la Unión Europea y los cultivos de cereal ascienden a un 21\% (EEA, 2010). En consecuencia, un gran número de especies silvestres, que en el caso de las aves se ha estimado en alrededor de un 50 \% (Pain y Pienkowski, 1997), dependen del manejo de los terrenos agrícolas. En el caso de España, el terreno agrícola y el dedicado al cereal suponen respectivamente valores próximos al 50\% y 25\% del territorio (Suárez, 2004; MAPAMA, 2020), por lo que estos hábitats son igualmente importantes para este grupo de vertebrados y en particular para las aves esteparias. De hecho, dada su superficie absoluta y el retraso de la intensificación agrícola respecto a una mayoría de países de Europa occidental, la importancia de España para estas aves es especialmente destacada en el conjunto de la UE, hasta el punto de que mantiene las mayores poblaciones de varias especies y porcentajes muy elevados de otras muchas (BirdLife Internacional, 2004; Suárez et al., 2009; Keller et al., 2020). Sin embargo, los procesos de intensificación, que en España se concentraron en las décadas de los años 60 y 70 (Suárez-Seoane et al., 2002; Suárez et al., 2009), han acabado por afectar igualmente a este grupo de aves, muchas de cuyas especies afrontan desde hace tiempo una situación de declive continuado, tal y como lo reflejan los programas de seguimiento a largo plazo de la Socie- 
dad Española de Ornitología (Programa SACRE: Carrascal y Palomino, 2008; Escandell y Escudero, 2020).

En este trabajo se exponen los cambios de la comunidad de aves en un área representativa de los cultivos cerealistas de secano del centro de España, censada en cuatro estaciones reproductivas entre 1984 y 2019. Los objetivos son:

1.- Verificar los cambios del paisaje agrícola en los 35 años del estudio.

2.- Analizar la evolución de la comunidad de aves a lo largo de la serie temporal estudiada.

3.- Analizar la distribución de las principales especies entre los distintos sustratos agrarios.

4.- Comprobar en qué medida los cambios de población detectados siguen las mismas tendencias que los registrados en España a escalas mayores.

Dada la escala local del estudio y el procedimiento de censo, el análisis se restringe principalmente al grupo de las aves paseriformes.

\section{Material y métodos}

\section{Área de estudio}

El área de estudio se sitúa en la provincia de Segovia, al sur de la localidad de Sepúlveda ( $\left.41^{\circ} 18^{\prime} \mathrm{N}, 3^{\circ} 55^{\prime} \mathrm{O}\right)$, a una altitud de unos 1000 msnm; el clima es mediterráneo continental (Font, 1983). La vegetación potencial es un encinar supramediterráneo (Quercus ilex), casi totalmente sustituido por cereales de secano (trigo y cebada, y algo de centeno), que conforman un paisaje agrícola de aspecto estepario, sin apenas elementos arbóreos y arbustivos y relieve predominantemente llano, característico de amplias áreas de las mesetas peninsulares (más detalles en Tellería et al., 1988).

El estudio se realizó en los años 1984, 2015, 2016 y 2019, en un sector de 750 ha en el que se diferenciaron ocho itinerarios lineales de una longitud aproximada de $1 \mathrm{~km}$ (total de 9,1 km). A partir de la información aportada por fotografías aéreas 1:18.000, se levantó en 1984 una plantilla esquemática de cada itinerario, con la red viaria y los límites entre parcelas agrícolas; las plantillas fueron actualizadas en 2015 con la información proporcionada por el Visor SigPac V 3.5 (www.sigpac.jcyl.es). Inmediatamente antes de la realización de los censos de aves, se visitó la zona de estudio para asignar el uso de cada una de las parcelas a uno de los siguientes sustratos:

(1) Pastos, cultivos abandonados o bien parcelas no cultivadas situadas en vaguadas, con suelos más profundos; por su parecido en cuanto al tipo de vegetación y por la dificultad de cuantificar su superficie, en este sustrato se incluyeron las lindes (caminos con vegetación ruderal, ribazos, acequias y límites no cultivados entre parcelas de cultivo). 
(2) Mies, parcelas sembradas con cereal de invierno, crecido en la época de censo, pero todavía verde (en general, falta más de un mes para la siega).

(3) Barbechos, parcelas en descanso tras la siega del año anterior.

(4) Arados, barbechos arados, en general destinados a siembra de girasol.

Para cada uno de estos sustratos se calculó la superficie en hectáreas para cada itinerario y año en una banda de $100 \mathrm{~m}$ de anchura (50 m a cada lado del itinerario); para las lindes, la longitud en metros.

Los censos de aves se realizaron entre mediados de mayo y principios de junio, en las tres primeras horas después del amanecer, con condiciones meteorológicas adecuadas; en 1984 se realizaron dos censos, por lo que se usó la media de las observaciones obtenidas. El método aplicado fue el transecto (Bibby et al., 2000), con una banda de recuento de $50 \mathrm{~m}$ a cada lado del observador, lo que permitió calcular índices de densidad para cada itinerario y sustrato agrario. Las aves registradas dentro de la banda de recuento se anotaron en las plantillas de referencia, en las parcelas en las que fueron observadas (no se anotaron las aves que cruzaron en vuelo los itinerarios); los aláudidos en canto se registraron cuando el centro del círculo de vuelo cayó dentro de la banda de recuento, y siempre que se levantaron dentro de la misma, independientemente de su comportamiento posterior. A partir de los censos se derivaron las siguientes variables por itinerario y año: densidad ( $n^{\circ}$ de aves/10 ha) para cada especie de ave registrada; densidad total, como la suma de las densidades parciales; riqueza, como número de especies distintas registradas; las variables de densidad se calcularon también para cada sustrato en el conjunto de los cuatro años. Salvo para la densidad total, tanto la estima de estas variables, como los análisis realizados, se restringieron a un total de 13 especies (los paseriformes, con excepción de la corneja negra). Los nombres científicos y comunes de las especies se dan en el Anexo.

Los cambios poblacionales registrados se compararon para las cuatro especies más abundantes con los estimados a partir de los datos del programa SACRE para distintos periodos (Seoane y Carrascal, 2007; Carrascal y Palomino, 2008; Escandell y Escudero, 2020). Para este estudio, se calcularon como la variación porcentual (positiva o negativa) para todo el periodo de 35 años (a partir de la diferencia en el número de individuos censados entre 1984 y 2019) y como porcentaje medio anual.

Bajo el supuesto de que los valores interanuales están ligados dentro de cada itinerario (en especial los de los tres últimos años), se utilizó el test de Friedman para muestras dependientes (Zar, 1984) para evaluar los cambios entre años de las variables aviares estimadas; solo se analizó la densidad de las especies con al menos 20 individuos censados en los cuatro años de estudio y presentes en al menos diez de los 32 itinerarios (ocho itinerarios por cuatro años). Los cuatro tests con resultado significativo ( $p$ " 0,05) se sometieron a evaluación posterior comparando 1984 con cada uno de los tres últi- 
mos años (2015, 2016 y 2019); dado que es razonable considerar los datos de 1984 como independientes, para estos análisis post-hoc se utilizó el test de la U de MannWhitney. Para el análisis de la asociación entre pares de variables se aplicó el coeficiente de correlación no paramétrico de Spearman. El uso de los sustratos agrarios por las especies de aves se comparó con la disponibilidad de los mismos mediante el índice de electividad de Ivlev, que varía entre -1 y +1 (máxima selección negativa y positiva, respectivamente), mientras los valores próximos a cero indican ausencia de selección (Atienza, 1994). De acuerdo con las recomendaciones de este autor, se ha considerado 0,5 como valor absoluto indicador de una selección clara. Dada la baja densidad y tendencia al gregarismo de bastantes especies, los índices se calcularon para el conjunto de los cuatro años.

\section{Resultados}

\section{Cambios en el paisaje agrícola}

La estructura del paisaje agrícola apenas ha variado en los 35 años del estudio, ya que no ha habido concentración parcelaria desde 1984; el cambio principal ha sido un pequeño aumento de la superficie de los pastos en los dos últimos años, atribuible al abandono de algunas parcelas cultivadas de pequeña extensión (Tabla 1). No obstante, hay notables diferencias entre itinerarios, evidenciadas por la variación de las superficies cubiertas por los distintos sustratos (Tabla 1), así como por las diferencias en el número (de 7 a 13) y tamaño medio (de 0,78 a 1,34 ha) de las parcelas agrarias, y por la cantidad lineal de lindes (correlación de Spearman entre esta variable y el número de parcelas: $r=0,740 ; p<0,05 ; n=8$ itinerarios; Fig. 1 ), rasgos que se han mantenido sin cambios apreciables en el periodo de estudio.

\begin{tabular}{|lcccc|}
\hline & $\begin{array}{c}\text { Pastos } \\
\text { (pasture) }\end{array}$ & $\begin{array}{c}\text { Barbechos } \\
\text { (fallow) }\end{array}$ & $\begin{array}{c}\text { Arados } \\
\text { (ploughed fields) }\end{array}$ & $\begin{array}{c}\text { Mies } \\
\text { (cornfields) }\end{array}$ \\
\hline 1984 & $4,4(0-1,8)$ & $4,7(0-1,5)$ & $26,7(0-6,6)$ & $55,0(5,2-9,2)$ \\
\hline 2015 & $4,4(0-2,3)$ & $7,2(0-3,4)$ & $38,8(0,2-8,1)$ & $40,4(1,8-7,8)$ \\
\hline 2016 & $5,2(0-2,3)$ & $1,3(0-1,4)$ & $29,2(1,3-6,9)$ & $55,1(2,5-9,5)$ \\
\hline 2019 & $6,5(0-3,7)$ & $6,5(0-4,7)$ & $30,3(0-10,7)$ & $47,5(1,9-10,8)$ \\
\hline$\%$ & 5,7 & 5,4 & 34,4 & 54,5 \\
\hline
\end{tabular}

Tabla 1.- Variación entre años de la superficie (ha) ocupada por cada uno de los sustratos agrarios (entre paréntesis, valores mínimo y máximo registrados en los ocho itinerarios); \%: porcentaje medio del área de censo (90,8 ha) cubierto por cada sustrato en el conjunto de los cuatro años.

Table 1.- Inter-annual variation in the area (ha) occupied by each of the agricultural substrates (in brackets, minimum and maximum values recorded in the eight routes); \%: average percentage of the census area ( 90.8 ha) covered by each substrate over the four years as a whole. 


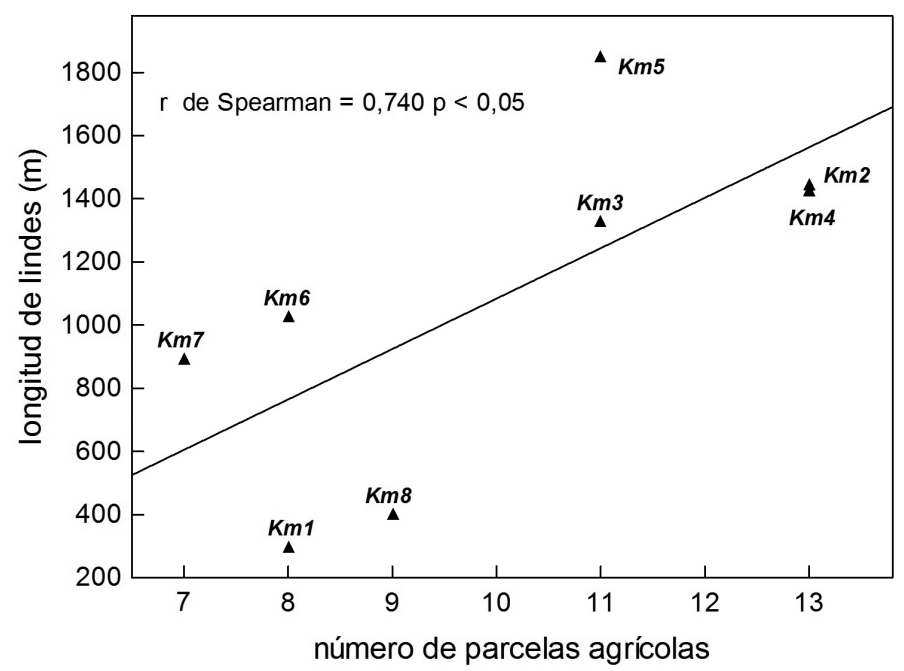

Fig. 1.- Relación entre el número de parcelas agrícolas y la longitud de lindes.

Fig. 1.- Relationship between the number of agricultural fields and the linear amount of field borders.

Los barbechos apenas representan un 5\% del área, por lo que arados y mieses, que cubren casi un $90 \%$ de la misma, han originado la mayor parte de los cambios interanuales en los usos agrarios. Los primeros han ocupado de un 30,9\% a un 44,9\% de la superficie cultivada y los segundos de un $46,8 \%$ a un $64,4 \%$

\section{Cambios en las comunidades de aves}

La comunidad de aves estuvo formada por 17 especies (14 de ellas paseriformes), de las que ocho fueron registradas todos los años y cuatro en un único año (Anexo). De las 13 especies analizadas (los paseriformes con exclusión de la corneja negra), los alaúdidos, con cuatro especies, sumaron dos tercios de la densidad total, y una de sus especies, la alondra común, un tercio; junto con el escribano triguero, otra especie típica de cultivos cerealistas, sumaron cerca del $80 \%$ de la comunidad (Tabla 2). Mientras la densidad total de aves no varió significativamente a lo largo del periodo, la riqueza media aumentó (Tabla 2), principalmente como resultado de una mayor distribución entre itinerarios de seis especies en los tres últimos años (la cogujada, la terrera, el triguero, la lavandera boyera, la tarabilla europea y el pardillo), mientras que la collalba gris fue la única con un descenso claro en su frecuencia de aparición (en cuatro itinerarios en 1984 y uno en los dos últimos años). Aunque los resultados mostraron una aparente tendencia al descenso de los alaúdidos, la única especie con una pérdida significativa de densidad fue la calandria común (Tabla 2, Fig. 2). Dos especies, el escribano triguero y la lavandera boyera, mostraron un incremento significativo de la densidad entre 1984 y los tres últimos años (Tabla 2, Fig. 2). 


\begin{tabular}{|c|c|c|c|c|c|c|c|c|c|c|}
\hline & fre & $\%$ & 1984 & 2015 & 2016 & 2019 & $\begin{array}{c}\text { Friedman } \\
\text { ANOVA }\end{array}$ & $\begin{array}{c}P-H \\
2015\end{array}$ & $\begin{array}{l}\text { P-H } \\
2016\end{array}$ & $\begin{array}{c}\text { P-H } \\
2019\end{array}$ \\
\hline Riqueza media & & & 3,4 & 5,6 & 6,1 & 5,4 & $\begin{aligned} x^{2} & =13,544 \\
p & =0,0040\end{aligned}$ & 0,020 & 0,002 & 0,015 \\
\hline Alondra común & 30 & 35,8 & 8,6 & 5,7 & 4,8 & 5,8 & $\begin{aligned} X^{2} & =6,987 \\
p & =0,072\end{aligned}$ & & & \\
\hline Calandria común & 29 & 18,8 & 5,2 & 3,5 & 2,8 & 1,6 & $\begin{aligned} x^{2} & =11,375 \\
p & =0,010\end{aligned}$ & 0,248 & 0,083 & 0,006 \\
\hline Terrera común & 22 & 9,3 & 1,0 & 1,1 & 2,0 & 2,4 & $\begin{aligned} x^{2} & =4,971 \\
p & =0,174\end{aligned}$ & & & \\
\hline Escribano triguero & 27 & 11,5 & 1,0 & 2,7 & 2,7 & 1,6 & $\begin{array}{c}x^{2}=13,458 \\
p=0,004\end{array}$ & 0,050 & 0,021 & 0,081 \\
\hline Pardillo común & 10 & 5,0 & 0,05 & 1,0 & 2,0 & 0,4 & $\begin{aligned} x^{2} & =5,930 \\
p & =0,115\end{aligned}$ & & & \\
\hline Lavandera boyera & 10 & 3,6 & 0,0 & 1,1 & 1,2 & 0,2 & $\begin{aligned} x^{2} & =10,647 \\
p & =0,014\end{aligned}$ & 0,065 & 0,004 & 0,317 \\
\hline Tarabilla europea & 12 & 5,1 & 0,05 & 2,0 & 0,7 & 0,8 & $\begin{aligned} x^{2} & =7,091 \\
p & =0,069\end{aligned}$ & & & \\
\hline Alaúdidos ${ }^{1}$ & 32 & 65,8 & 14,8 & 10,6 & 10,0 & 10,4 & $\begin{aligned} x^{2} & =5,654 \\
p & =0,130\end{aligned}$ & & & \\
\hline Fringílidos ${ }^{2}$ & 12 & 10,4 & 0,05 & 2,7 & 3,0 & 1,5 & $\begin{aligned} X^{2} & =4,860 \\
p & =0,182\end{aligned}$ & & & \\
\hline Densidad total & & & 17,1 & 19,8 & 17,7 & 15,2 & $\begin{aligned} X^{2} & =3,684 \\
p & =0,298\end{aligned}$ & & & \\
\hline
\end{tabular}

${ }^{1}$ cogujada común, alondra, calandria y terrera; ${ }^{2}$ pardillo y jilguero europeo.

Tabla 2.- Variación interanual ( $\mathrm{n}=8$ itinerarios) de la riqueza media de aves, y de las densidades medias $\left(n^{\circ}\right.$ de aves/10 ha) de las especies con al menos 20 individuos censados en los cuatro años de estudio y presentes en al menos 10 itinerarios; fre: frecuencia de aparición sobre un total de 32 censos (4 años por 8 itinerarios); \%: porcentaje de la densidad total de cada especie y familia (alaúdidos y fringílidos) para el conjunto de los cuatro años. Friedman: resultados del test de Friedman $(n=8, g l=3)$ realizado para comparar los valores de densidad y riqueza entre años (diferencias significativas en negrita). P-H: resultado de las comparaciones post-hoc entre 1984 y los tres años restantes.

Table 2.- Inter-annual variation ( $\mathrm{n}=8$ routes) in mean bird richness, and mean densities ( $\mathrm{n}^{\circ}$ of birds $/ 10$ ha) of species with at least 20 individuals censused in the four years of the study and present in at least 10 routes; fre: frequency of occurrence over a total of 32 censuses (4 years for 8 routes); \%: percentage of the total density of each species and family (larks and fringilids) for the four years as a whole. Friedman: Friedman tests $(n=8, g l=3$ ) carried out to compare density and richness values between years (significant differences in bold). P-H: post-hoc tests between 1984 and the remaining three years.

El índice de Ivlev tomó valores positivos y en general altos para los pastos, que fueron seleccionados muy por encima de su disponibilidad por una mayoría de especies, así como por el conjunto de la comunidad (Tabla 3). En el resto de sustratos dominaron los valores negativos o neutros (próximos a cero), salvo la selección positiva de los arados por la terrera común y de los barbechos por el pardillo común. 

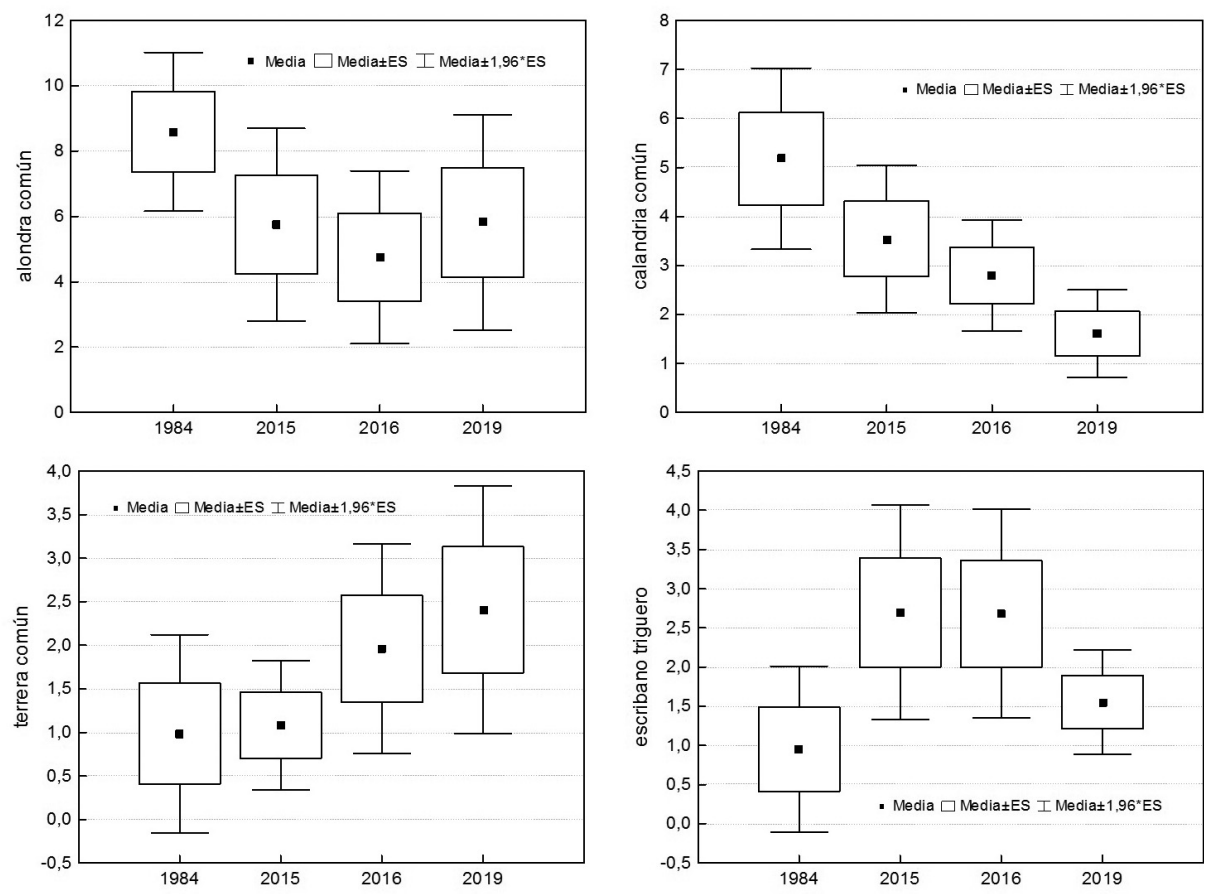

Fig. 2.- Variación interanual de la densidad media ( $n^{\circ}$ de aves/10 ha) de las especies más abundantes.

Fig. 2.- Inter-annual variation in the mean density (birds/10 ha) of the most abundant species.

\begin{tabular}{|lcccccccc|}
\hline & $\begin{array}{c}\text { Alondra } \\
\text { común }\end{array}$ & $\begin{array}{c}\text { Calandria } \\
\text { común }\end{array}$ & $\begin{array}{c}\text { Terrera } \\
\text { común }\end{array}$ & $\begin{array}{c}\text { Lavandera } \\
\text { boyera }\end{array}$ & $\begin{array}{c}\text { Tarabilla } \\
\text { europea }\end{array}$ & $\begin{array}{c}\text { Pardillo } \\
\text { común }\end{array}$ & $\begin{array}{c}\text { Escribano } \\
\text { triguero }\end{array}$ & Total \\
\hline Pastos & 0,57 & 0,35 & 0,10 & 0,59 & 0,87 & 0,82 & 0,85 & 0,69 \\
\hline Barbechos & 0,03 & 0,19 & $-0,22$ & $-1,00$ & $-1,00$ & 0,41 & $-0,76$ & $-0,08$ \\
\hline Arados & $-0,29$ & $-0,01$ & 0,37 & $-0,43$ & $-1,00$ & $-0,36$ & $-0,65$ & $-0,13$ \\
\hline Mies & $-0,04$ & $-0,10$ & $-0,57$ & 0,05 & $-1,00$ & $-1,00$ & $-0,83$ & $-0,26$ \\
\hline
\end{tabular}

Tabla 3.- Valores del índice de Ivlev para las especies con al menos 20 individuos censados en los cuatro años de estudio y presentes en al menos 10 de 32 itinerarios, y para el conjunto de la comunidad; en negrita, valores absolutos mayores de 0,5.

Table 3.- Ivlev index values for species with at least 20 individuals censused in the four years of study and present in at least 10 out of 32 routes, and for the community as a whole; in bold, absolute values greater than 0.5 .

Las tendencias declinantes en el área de estudio de la alondra y la calandria coinciden con las apuntadas para toda España por el programa SACRE; sin embargo, la tendencia al incremento de la terrera común y el escribano triguero no encontraron correspondencia a escala nacional, en la que estas dos especies no muestran una tendencia clara (Tabla 4). 


\begin{tabular}{|l|c|c|c|c|c|}
\hline & $1996-2004^{\mathrm{a}}$ & $1998-2006^{\mathrm{b}}$ & $1998-2019^{c}$ & \multicolumn{2}{|c|}{ Este estudio } \\
\cline { 2 - 6 } & $\begin{array}{c}\text { Tasa de } \\
\text { cambio }\end{array}$ & $\begin{array}{c}\text { \% cambio } \\
\text { anual }\end{array}$ & $\begin{array}{c}\text { España/medit. } \\
\text { norte }\end{array}$ & $\begin{array}{c}\text { \% cambio } \\
\text { anual }\end{array}$ & $\begin{array}{c}\% 5 \\
\text { años }\end{array}$ \\
\hline Alondra común & $-0,02^{*}$ & $-5,0$ (decreciente) & - moderada/-moderada & $-0,96$ & $-33,8$ \\
\hline Calandria común & $-0,02^{*}$ & $-2,8$ (decreciente) & - -moderada/-moderada & $-1,95$ & $-68,4$ \\
\hline Terrera común & $-0,03$ n.s. & $-4,2$ (decreciente) & +moderada/no estimada & 6,3 & 221,1 \\
\hline Escribano triguero & 0 n.s. & $-2,7$ (decreciente) & -moderada/estable & 5 & 175 \\
\hline
\end{tabular}

a Seoane y Carrascal (2007). ${ }^{b}$ Carrascal y Palomino (2008). CSACRE: Escandell y Escudero (2020), para toda España y la región mediterránea norte.

Tabla 4.- Tendencias de población según diversas fuentes de las cuatro especies más abundantes y distribuidas en el área de estudio. Tasa de cambio: pendiente de la regresión; *: p<0,05; n.s.: no significativa. Tendencias de cambio: decreciente, estable, moderada (+/-: creciente/decreciente).

Table 4.- Population trends for the four most abundant and widespread species in the study area. Rate of change: slope of regression; *: $p<0.05$; n.s.: not significant. Change trends: decreasing, stable, moderate (+/-: increasing/decreasing).

\section{Discusión}

Acorde con los procesos de intensificación del agro español (concentración parcelaria, disminución del barbecho, incremento de abonos inorgánicos, etc.), ocurridos principalmente en las décadas de 1960 y 1970 (Suárez et al., 2009), el paisaje estudiado corresponde a una estepa cerealista intensificada. De hecho, el paisaje ya estaba intensificado en 1984, como lo demuestra la escasa superficie de pastos y de barbechos primaverales, de modo que los únicos cambios interanuales de cierta envergadura se deben a la variación alterna entre los dos usos agrarios dominantes, arados y mies.

Teniendo en cuenta que la escala del estudio solo es adecuada para caracterizar el grupo de las aves paseriformes, la comunidad registrada puede considerarse como genuinamente esteparia dado el dominio de los alaúdidos y el escribano triguero (de Juana, 1989). Faltan, sin embargo, algunas especies típicas de pastizales extensos y sub-estepas arbustivas (eriales antiguos y matorrales degradados; Tellería et al., 1988, Suárez et al., 2009), hábitats ausentes en el área debido a su alto grado de intensificación agrícola. Aunque la comparación con otros estudios que han usado la misma metodología de censo tiene limitaciones evidentes (por causas como la ausencia en éste de los hábitats semi-naturales citados, la diferente proporción de los sustratos agrarios, o la escala geográfica, de comarcal a nacional), las densidades obtenidas (Tabla 2) caen en buena medida dentro del marco de variación registrado para las especies más extendidas: entre 1,3 y 4,7 aves/10 ha para la alondra común, de 4,4 a 13,5 para la calandria común, 0,9 a 3,1 para la terrera común y 1,1 a 3,2 para el escribano triguero (Tellería et al., 1988; Díaz et al., 1993; Suárez et al. 2002, 2003; Santos y Suárez, 2005). Los resultados no reflejan cambios significativos en la abundancia 
total de aves, mientras que se produce un aumento de la riqueza media de especies a lo largo de los años que puede explicarse por el incremento de varias especies en el periodo 2015-2019 (Anexo), incluyendo dos típicamente esteparias (la terrera común y el escribano triguero), acompañado de una mayor distribución entre itinerarios de todas ellas.

Limitaciones parecidas se encuentran al comparar la selección de sustratos agrarios con la mostrada por otros estudios, en especial por las diferencias de escala y por la disponibilidad relativa de los mismos (Suárez et al., 2009). Los resultados obtenidos, que muestran una preferencia clara por los pastos compartida por una mayoría de especies, corroboran los registrados por Suárez et al. (2009) en su revisión de los alaúdidos españoles, en áreas geográficas mucho mayores y hábitats mucho más variados. La relevancia de este sustrato a la escala del estudio es, por lo demás, obvia si se tiene en cuenta que apenas representa un 5\% del área, mientras que acumula un $37,7 \%$ de las aves contactadas y mantiene densidades casi diez veces mayores que el resto de los sustratos (Anexo). Los resultados de Tellería et al. (1988), obtenidos también en la comarca de Sepúlveda, aunque en un área mucho mayor y con una presencia elevada de eriales, muestran una selección positiva de los pastos por la alondra común, la calandria común, el escribano triguero y el pardillo común. Así mismo, revelan que esta última especie rechaza los barbechos, mientras que la terrera común los selecciona positivamente en vez de los arados. En conjunto, los barbechos se seleccionan por tres de estas cinco especies, una diferencia con el presente estudio que podría explicarse por la distinta escala y proporción de sustratos, pero posiblemente también por una tendencia creciente a labrar los barbechos entre la cosecha y el nuevo cultivo, con la consiguiente pérdida de recursos tróficos (Suárez et al., 2009). Los resultados obtenidos en otras localidades, confirman la selección positiva de los pastos ("herbazales") por la alondra y la calandria común sobre un conjunto de diez hábitats en la Rioja Alavesa (Gainzarain, 1996), mientras que las revisiones a escala peninsular de Suárez (2004) y Suárez et al. (2009) revelan de nuevo una preferencia de la terrera común por los barbechos, y de la alondra común y el escribano triguero por los pastos en paisajes dominados por el uso agrícola. Salvando las diferencias de método y ubicación geográfica, resultados equiparables se han obtenido en paisajes agrícolas de Portugal para el escribano triguero y la calandria común (Moreira, 1999; Morgado et al., 2010), y en Gran Bretaña para la alondra común (Donald et al., 2001a). La predilección de los pastos por las tres especies menos distribuidas (Tabla 3), se ajusta a lo esperado (Borrás y Senar, 2003; Illera, 2003), de modo que todas (tarabilla europea) o la gran mayoría de las observaciones (pardillo común) se concentran en los pastos y lindes; en el caso de la lavandera boyera, un ave asociada a los pastos húmedos en la meseta norte (Pérez-Tris, 2003), su respuesta neutra a la mies explica el elevado número de contactos en este sustrato mayoritario, localizados salvo excepciones muy cerca de acequias y lindes de juncos (obs. pers.). 
La predilección general por los pastos queda, sin embargo, muy matizada si se considera la proporción de aves observadas en las lindes (diferenciadas en los censos); así, para el conjunto de la comunidad, 99 de los 239 contactos asignados a pastos fueron registrados en las lindes, un sustrato dominado también por vegetación herbácea espontánea. Estos resultados resaltan la extraordinaria importancia que para las comunidades estudiadas tienen elementos del paisaje agrícola muy minoritarios pero estables, tanto en términos de densidad como de riqueza de especies (10 de 17 fueron detectadas en las lindes y 12 en los pastos; Anexo). Este hecho parece general en los ecosistemas agrícolas, donde estos elementos aumentan de modo notable la diversidad del paisaje y su oferta de recursos, tanto para las aves (semillas y artrópodos) como para otros organismos animales (Tellería et al., 1988; Vickery et al., 2009; Hof y Bright, 2010; Bartual et al., 2019). En todo caso, a la escala y configuración paisajística del área de estudio, en la que lindes y pastos representan poco más del $5 \%$, parece evidente que la combinación de estos dos sustratos, presentes en todos los itinerarios en el caso de las lindes, tienen efectos desproporcionadamente altos para el conjunto de la comunidad aviar.

La disminución de las dos especies más abundantes y extendidas en el área, la alondra y la calandria común, concuerda con los resultados del SACRE para toda España (Tabla 4). A escala regional, Gainzarain y Belamendia (2015) registran en la Rioja Alavesa tasas medias de variación anual de $-13,1 \%$ para la calandria común y 5,1\% para la terrera común, en la línea de estos resultados. Dado que apenas ha habido cambios en los sustratos agrarios y la estructura del paisaje no ha variado, los descensos registrados podrían deberse a factores externos al área de estudio actuando a una escala mayor, como una mejora del hábitat en áreas más o menos próximas (Suárez et al., 2009), o bien a otros que no se han tenido en cuenta, como un hipotético incremento en el uso de herbicidas e insecticidas (Vickery et al., 2009; Geiger et al., 2010; Mineau y Whiteside, 2013). En todo caso, dado el largo intervalo transcurrido entre censos, y la frecuencia de fluctuaciones interanuales en muchas especies de vertebrados (McCain et al., 2016), conviene tomar estos resultados con reserva.

Ya que el área de estudio ha sido totalmente intensificada, cabe esperar como escenario futuro más probable un mantenimiento de la estructura del paisaje agrícola, salvo que se den cambios relevantes en la política agraria europea (PAC) orientados a potenciar la eficacia de las medidas agro-ambientales (Llusia y Oñate, 2005; Concepción y Díaz, 2019; Šumrada et al., 2020). En este escenario, las poblaciones de alondra y calandria seguirían disminuyendo de mantenerse la deriva de las poblaciones ibéricas, mientras que es difícil hacer predicciones sobre el resto de las especies. Nuestros resultados, y los obtenidos por otros autores a escalas espaciales más extensas (Suárez, 2004; McMahon et al., 2010; Martin et al., 2019; Broughton et al., 2021; Díaz et al., 2021), muestran la relevancia de una diversidad agrícola elevada, con especial atención a la presencia de elementos estables, para mantener comunidades de aves densas 
y diversas en ecosistemas agrícolas intensificados. Por tanto, se recomienda respetar o aumentar las superficies de pasto e impulsar una estrategia de recuperación de lindes y ribazos con vegetación herbácea para favorecer a las numerosas especies que nidifican o buscan su alimento en estos ecotonos agrícolas (Buner et al., 2005; Gaüzère et al., 2019; Martin et al., 2019; Broughton et al., 2021).

\section{Agradecimientos}

El autor agradece las críticas de José Antonio Gainzarain y de un revisor anónimo, que contribuyeron a una mejora notable del trabajo. Los censos de 1984 fueron sufragados por el Proyecto n CAICYT 1429-82.

\section{Bibliografía}

Atienza, J.C., 1994. La utilización de índices en el estudio de la selección de recursos. Ardeola 41, 173-175.

Bartual, A.M., Sutter, L., Bocci, G., Moonen, A.-C., Cresswell, J., Entling, M., Giffard, B., Jacot, K., Jeanneret, P., Holland, J., Pfister, S., Pinter, O., Veromann, E., Winkler, K., Albrecht, M., 2019. The potential of different semi-natural habitats to sustain pollinators and natural enemies in European agricultural landscapes. Agriculture, Ecosystems and Environment $279,43-52$.

Benton, T.G., Vickery, J.A., Wilson, J. D., 2003. Farmland biodiversity: Is habitat heterogeneity the key? Trends in Ecology and Evolution 18, 182-188.

Bernis, F., 1972. La avifauna ibérica. Pasado, presente y futuro de las aves españolas. En: Fitter, R., Fernández-Cruz, M. (Eds.), El libro de las aves españolas, 6-16. Selecciones del Reader’s Digest (Ibérica), Madrid.

Bibby, C.J., Burgess, N.D., Hill, D.A., 2000. Bird census techniques. 2nd Edition. Academic Press, London.

BirdLife Internacional, 2004. Birds in Europe: population estimates, trends and conservation status. BirdLife Internacional, Cambridge.

Borrás, A., Senar, J.C., 2003. Pardillo común Carduelis cannabina. En: Martí, R., del Moral, J.C. (Eds.), Atlas de las Aves Reproductoras de España, 586-587. Dirección General de Conservación de la Naturaleza-Sociedad Española de Ornitología, Madrid.

Broughton, R.K., Chetcuti, J., Burgess, M.D., Gerard, F.F., Pywell, R.F., 2021. A regionalscale study of associations between farmland birds and linear woody networks of hedgerows and trees. Agriculture, Ecosystems and Environment 310, https://doi.org/10.1016/j.agee.2021.107300. 
Buner, F., Jenny, M., Zbinden, N., Naef-Daenzer, B., 2005. Ecologically enhanced areas - a key habitat structure for re-introduced grey partridges Perdix perdix. Biological Conservation 124, 373-381.

Carrascal, L.M., Palomino, D., 2008. Las aves comunes reproductoras en España. Población en 2004-2006. SEO/BirdLife, Madrid.

Concepción, E.D., Díaz, M., 2019. Varying potential of conservation tools of the Common Agricultural Policy for farmland bird preservation. Science of the Total Environment 694, https://doi.org/10.1016/j.scitotenv.2019.133618.

De Juana, E., 1989. Las aves esteparias en España. En: Seminario sobre zonas áridas en España, 199-221. Real Academia de Ciencias Exactas, Físicas y Naturales, Madrid.

Díaz, M., Concepción, E.D., Morales, M.B., Alonso, J.C., Azcárate, F.M., Bartomeus, I., Bota, G., Brotons, L., García, D., Giralt, D., Gutiérrez, J.E., López-Bao, J.V., Mañosa, S., Milla, R., Miñarro, M., Navarro, N., Olea, P.P., Palacín, C., Peco, B., Rey, P.J., Seoane, J., Suárez-Seoane, S., Schöb, C., Tarjuelo, R., Traba, J., Valera, F., Velado-Alonso, E., 2021. Environmental objectives of Spanish agriculture: scientific guidelines for their effective implementation under the Common Agricultural Policy 2023-2030. Ardeola 68, 445-460.

Díaz, M., Naveso, M.A., Rebollo, E., 1993. Respuestas de las comunidades nidificantes de aves a la intensificación agrícola en cultivos cerealistas de la Meseta Norte (Valladolid-Palencia, España). Aegypius 11, 1-6.

Donald, P.F., Evans, A.D., Buckingham, D.L., Muirhead, L.B., Wilson, J.D., 2001a. Factors affecting the territory distribution of skylarks Alauda arvensis breeding on lowland farmland. Bird Study 48, 271-278.

Donald, P.F., Green, R.E., Heath, M.F., 2001b. Agricultural intensification and the collapse of Europe's farmland bird populations. Proceedings of the Royal Society B: Biological Sciences 268, 25-29.

EEA, 2010. Assessing biodiversity in Europe — the 2010 report. European Environment Agency, Copenhagen.

Escandell, V, Escudero, E., 2020. Programa SACRE. En: Programas de seguimiento y grupos de trabajo de SEO/BirdLife 2019, 6-11. SEO/BirdLife, Madrid.

European Commission, 2020. Evaluation of the impact of the CAP on habitats, landscapes, biodiversity. Directorate-General for Agriculture and Rural Development 2019, Luxemburgo.

Font, I., 1983. Climatología de España y Portugal. Instituto Nacional de Meteorología, Madrid.

Gainzarain, J.A., 1996. Selección de hábitat de la avifauna en una comarca agrícola del Alto Valle del Ebro (Norte de España). Munibe, Cienc. nat. 48, 3-16.

Gainzarain, J.A., Belamendia, G., 2015. Las aves de distribución mediterránea en el País Vasco: abundancia y tendencia poblacional en el sur de Álava. Munibe, Cienc. nat. 63, 7-28. 
Gaüzère, P., Barbaro, L., Calatayud, F., Princé, K., Devictor, V., Raison, L., Sirami, C., Balent, G., 2019. Long-term effects of combined land-use and climate changes on local bird communities in mosaic agricultural landscapes. Agriculture, Ecosystems and Environment 289, https:/doi.org/10.1016/j.agee.2019.106722.

Geiger, F., Bengtsson, J., Berendse, F., Weisser, W.W., Emmerson, M., Morales, M.B., Ceryngier, P., Liira, J., Tscharntke, T., Winqvist, C., Eggers, S., Bommarco, R., Pärt, T., Bretagnolle, V., Plantegenest, M., Clement, L.W., Dennis, C., Palmer, C., Oñate, J.J., Guerrero, I., Hawro, V., Aavik, T., Thies, C., Flohre, A., Hänke, S., Fischer, C., Goedhart, P.W., Inchausti, P., 2010. Persistent negative effects of pesticides on biodiversity and biological control potential on European farmland. Basic and Applied Ecology 11, 97-105.

Gregory, R.D., van Strien, A., Voříšek, P., Meyling, A.W.G., Noble, D.G., Foppen, R.P.B., Gibbons, D.W., 2005. Developing indicators for European birds. Philosophical Transactions of the Royal Society B: Biological Sciences 360, 269-288.

Hof, A.R., Bright, P.W., 2010. The impact of grassy field margins on macro-invertebrate abundance in adjacent arable fields. Agriculture, Ecosystems and Environment 139, 280 283.

Illera, J.C., 2003. Tarabilla común Saxicola torquata. En: Martí, R., del Moral, J.C. (Eds.), Atlas de las Aves Reproductoras de España, 430-431. Dirección General de Conservación de la Naturaleza-Sociedad Española de Ornitología, Madrid.

Keller, V., Herrando, S., Voříšek, P., Franch, M., Kipson, M., Milanesi, P., Martí, D., Anton, M., Klvaňová, A., Kalyakin, M.V., Bauer, H.-G., Foppen, R.P.B., 2020. European Breeding Bird Atlas 2: Distribution, Abundance and Change. European Bird Census Council \& Lynx Edicions, Barcelona.

Llusia, D., Oñate, J.J., 2005. Are the conservation requirements of pseudo-steppe birds adequately covered by Spanish agri-environmental schemes? An ex-ante assessment. Ardeola $52,31-42$

Madroño, A., González, C., Atienza, J.C., 2004. Libro Rojo de las Aves de España. Dirección General para la Biodiversidad-SEO/BirdLife, Madrid.

MAPAMA, 2020. Anuario de estadística 2019. Ministerio de Agricultura y Pesca, Alimentación y Medio Ambiente, Madrid.

Martin, A.E., Collins, S.J., Crowe, S., Girard, J., Naujokaitis-Lewis, I., Smith, A.C., Lindsay, K., Mitchell, S., Fahrig, L., 2019. Effects of farmland heterogeneity on biodiversity are similar to-or even larger than-the effects of farming practices. Agriculture, Ecosystems and Environment 288, doi.org/10.1016/j.agee.2019.106698.

McCain, C., Szewczyk, T., Bracy Knight, K., 2016. Population variability complicates the accurate detection of climate change responses. Global Change Biology 22, 2081 2093. 
McMahon, B. J., Giralt, D., Raurell, M., Brotons, L., Bota, G., 2010. Identifying set-aside features for bird conservation and management in northeast Iberian pseudo-steppes. Bird Study 57, 289-300.

Mineau, P., Whiteside, M., 2013. Pesticide acute toxicity is a better correlate of U.S. grassland bird declines than agricultural intensification. PLoSONE 8(2): e57457. https://doi.org/10.1371/journal.pone.0057457.

Moreira, F., 1999. Relationships between vegetation structure and breeding bird densities in fallow cereal steppes in Castro Verde, Portugal. Bird Study 46, 309-318.

Morgado, R., Beja, P., Reino, L., Gordinho, L., Delgado, A., Borralho, R., Moreira, F., 2010. Calandra lark habitat selection: Strong fragmentation effects in a grassland specialist. Acta Oecologica 36, 63-73.

Pain, D.J., Pienkowski, M.W. (Eds.), 1997. Farming and birds in Europe: the common agricultural policy and its implications for bird conservation. Academic Press, London.

Pérez-Tris, J., 2003. Lavandera boyera Motacilla flava. En: Martí, R., del Moral, J.C. (Eds.), Atlas de las Aves Reproductoras de España, 398-399. Dirección General de Conservación de la Naturaleza-Sociedad Española de Ornitología, Madrid.

Robinson, R.A., Sutherland, W.J., 2002. Post-war changes in arable farming and biodiversity in Great Britain. Journal of Applied Ecology 39, 157-176.

Santos, T., Suárez, F., 2005. Biogeography and population trends of Iberian steppe birds. In: Bota, G., Morales, M.B., Mañosa, S., Camprodon, J. (Eds.), Ecology and conservation of steppe-land birds, 69-102. Lynx Edicions \& Centre Tecnològic Forestal de Catalunya, Barcelona.

Seoane, J., Carrascal, L.M., 2007. Interspecific differences in population trends of Spanish birds are related to habitat and climatic preferences. Global Ecology and Biogeography, https://doi.org/10.1111/j.1466-8238.2007.00351. x.

Suárez, F., 2004. Aves y agricultura en España peninsular: Una revisión sobre el estado actual de conocimiento y una previsión sobre su futuro. En, Tellería, J.L. (Ed.), La Ornitología hoy: Homenaje al profesor Francisco Bernis Madrazo, 223-265. Editorial Complutense, Madrid.

Suárez, F., Garza, V., Morales, M.B., 2002. Habitat use of the two sibling species, the Shorttoed Calandrella brachydactyla and the Lesser Short-toed Calandrella rufescens Larks, in mainland Spain. Ardeola 49, 259-272.

Suárez, F., Garza, V., Morales, M.B., 2003. The role of extensive cereal crops, dry pasture and shrub-steppe in determining Sky-lark Alauda arvensis densities in the Iberian Peninsula. Agriculture, Ecosystems and Environment 95, 551-557.

Suárez, F., Hervás, I., Herranz, J., 2009. Las alondras de España peninsular. Dirección General para la Biodiversidad, Ministerio de Medio Ambiente y Medio Rural y Marino, Madrid. 
Suárez-Seoane, S., Osborne, P.E., Baudry, J., 2002. Responses of birds of different biogeographic origins and habitat requirements to agricultural land abandonment in northern Spain. Biological Conservation 105, 333-344.

Šumrada, T., Kmecl, P., Erjavec, E., 2020. Do the EU's Common agricultural policy funds negatively affect the diversity of farmland birds? Evidence from Slovenia. Agriculture, Ecosystems and Environment 306, https://doi.org/10.1016/j.agee.2020.107200.

Tellería, J.L., Santos, T., Álvarez, G., Sáez-Royuela, C., 1988. Avifauna de los campos de cereales del interior de España. En: Bernis, F. (Ed.), Aves de los medios urbano y agrícola en las mesetas españolas. Monografías 2, 173-319. Sociedad Española de Ornitología, Madrid.

Tucker, G.M., Heath, M.F., 1994. Birds in Europe: Their Conservation Status. BirdLife International, Cambridge.

Vickery, J.A., Feber, R.E., Fuller, R.J., 2009. Arable field margins managed for biodiversity conservation: A review of food resource provision for farmland birds. Agriculture, Ecosystems and Environment 133, 1-13.

Zar, J.H., 1984. Biostatistical Analysis. Second Edition. Prentice-Hall, London.

Fecha de recepción/ Date of reception: 25/10/2021

Fecha de aceptación / Date of acceptance: 01/12/2021

Editor Asociado / Associate editor: Iván de la Hera 
Anexo. Composición de la comunidad de aves. Se indica para cada especie el número de aves por año (los datos de 1984 son la media de dos censos) y la distribución de densidades (aves/10 ha) según sustratos agrarios para el conjunto de los cuatro años de estudio. P: pastos, B: barbechos, A: arados, M: mies.

Annexe. Composition of the bird community. The number of birds per year is given for each species (1984 data are the average of two censuses) and the distribution of densities (birds/10 ha) according to agricultural substrates for the four years of the study. P: pasture, B: fallow, A: ploughed fields, M: cornfield.

\begin{tabular}{|c|c|c|c|c|c|c|c|c|c|}
\hline & 1984 & 2015 & 2016 & 2019 & Total & $P$ & B & A & $M$ \\
\hline $\begin{array}{l}\text { Codorniz común } \\
\text { Coturnix coturnix (Linnaeus, 1758) }\end{array}$ & 0,5 & 2 & 2 & 4 & 8,5 & 0,78 & 0,00 & 0,00 & 0,33 \\
\hline $\begin{array}{l}\text { Perdiz roja } \\
\text { Alectoris rufa (Linnaeus, 1758) }\end{array}$ & 2 & 0 & 0 & 0 & 2 & 0,00 & 0,00 & 0,00 & 0,10 \\
\hline $\begin{array}{l}\text { Paloma bravía } \\
\text { Columba livia (Gmelin, 1789) }\end{array}$ & 0 & 0 & 0 & 2 & 2 & 0,00 & 0,00 & 0,16 & 0,00 \\
\hline $\begin{array}{l}\text { Cogujada común } \\
\text { Galerida cristata (Linnaeus, 1758) }\end{array}$ & 0 & 2 & 4 & 5 & 11 & 0,00 & 0,00 & 0,72 & 0,10 \\
\hline $\begin{array}{l}\text { Alondra común } \\
\text { Alauda arvensis (Linnaeus, 1758) }\end{array}$ & 77 & 51 & 41 & 51 & 220 & 21,78 & 6,33 & 3,32 & 5,56 \\
\hline $\begin{array}{l}\text { Calandria común } \\
\text { Melanocorypha calandra (Linnaeus, 1766) }\end{array}$ & 47,5 & 32 & 26 & 15 & 120,5 & 6,81 & 4,81 & 3,20 & 2,70 \\
\hline $\begin{array}{l}\text { Terrera común } \\
\text { Calandrella brachydactyla (Leisler, 1814) }\end{array}$ & 9,5 & 10 & 18 & 21 & 58,5 & 1,94 & 1,01 & 3,44 & 0,43 \\
\hline $\begin{array}{l}\text { Lavandera boyera } \\
\text { Motacilla flava (Linnaeus, 1758) }\end{array}$ & 0 & 9 & 11 & 2 & 22 & 2,33 & 0,00 & 0,24 & 0,66 \\
\hline $\begin{array}{l}\text { Estornino negro } \\
\text { Sturnus unicolor (Temminck, 1820) }\end{array}$ & 3,5 & 3 & 0 & 1 & 7,5 & 0,00 & 0,00 & 0,60 & 0,00 \\
\hline $\begin{array}{l}\text { Tarabilla europea } \\
\text { Saxicola rubicola (Linnaeus, 1766) }\end{array}$ & 0,5 & 17 & 7 & 7 & 31,5 & 12,25 & 0,00 & 0,00 & 0,00 \\
\hline $\begin{array}{l}\text { Tarabilla norteña } \\
\text { Saxicola rubetra (Linnaeus, 1758) }\end{array}$ & 1 & 0 & 0 & 0 & 1 & 0,39 & 0,00 & 0,00 & 0,00 \\
\hline $\begin{array}{l}\text { Collalba gris } \\
\text { Oenanthe oenanthe (Linnaeus, 1758) }\end{array}$ & 2,5 & 3 & 1 & 1 & 7,5 & 2,33 & 0,25 & 0.08 & 0,00 \\
\hline $\begin{array}{l}\text { Jilguero europeo } \\
\text { Carduelis carduelis (Linnaeus, 1758) }\end{array}$ & 0 & 16 & 9 & 9 & 34 & 10,11 & 0,00 & 0,64 & 0,00 \\
\hline $\begin{array}{l}\text { Pardillo común } \\
\text { Linaria cannabina (Linnaeus, 1758) }\end{array}$ & 0,5 & 9 & 18 & 4 & 31,5 & 8,75 & 2,03 & 0,40 & 0,00 \\
\hline $\begin{array}{l}\text { Corneja negra } \\
\text { Corvus corone (Linnaeus, 1758) }\end{array}$ & 2 & 0 & 0 & 0 & 2 & 0,00 & 0,00 & 0,00 & 0,10 \\
\hline $\begin{array}{l}\text { Gorrión molinero } \\
\text { Passer montanus (Linnaeus, 1758) }\end{array}$ & 1 & 2 & 0 & 2 & 5 & 1,75 & 0,00 & 0,00 & 0,025 \\
\hline $\begin{array}{l}\text { Escribano triguero } \\
\text { Emberiza calandra (Linnaeus, 1758) }\end{array}$ & 8 & 24 & 24 & 14 & 70 & 23,73 & 0,25 & 0,40 & 0,18 \\
\hline Número de aves & 155,5 & 180 & 161 & 138 & 634,5 & 239 & 29 & 165 & 201,5 \\
\hline Densidad ( $n^{\circ}$ de aves/10 ha) & 17,1 & 19,8 & 17,7 & 15,2 & & 116,58 & 14,72 & 13,20 & 10,18 \\
\hline Riqueza total & 13 & 13 & 11 & 13 & 17 & 12 & 6 & 11 & 10 \\
\hline $\begin{array}{l}\text { Idem de Paseriformes } \\
\text { (excluyendo la corneja negra) }\end{array}$ & 10 & 11 & 10 & 12 & 13 & 11 & 6 & 10 & 7 \\
\hline
\end{tabular}

